\title{
NANOSPRAY PROCESSING OF SILVER NANOPARTICLES FOR FORMATION OF DRIED DEPOSITS
}

\author{
'Anna TYCOVA, ${ }^{1}$ Vladimir JONAS, ${ }^{1} J a n$ PRIKRYL, ${ }^{2}$ Adela KOTZIANOVA, ${ }^{2}$ Vladimir VELEBNY, \\ ${ }^{1}$ Frantisek FORET \\ 1Institute of Analytical Chemistry of the CAS, v.v. i., Brno, EU, Czech Republic,tycova@iach.cz \\ ${ }^{2}$ Contipro a.s., R\&D Department, Dolni Dobrouc, EU, Czech Republic, adela.kotzianova@contipro.com
}

https://doi.org/10.37904/nanocon.2020.3768

\begin{abstract}
Nanospray transfers liquid into an aerosol via electrostatic forces created between an emitter and a counter electrode. Herein, we present compact laboratory-made instrumentation for nanospray-processing of silver colloid. The instrumentation is based on the pressurized polysulfone chamber fixing a disposable vial with an immersed platinum electrode and a long fused silica capillary supplying the sprayed liquid to the emitter. The combination of low flow rates $(70 \mathrm{~nL} / \mathrm{min})$ with a sharp emitter, fabricated on a 3D printed grinding station, resulted in a fine aerosol. The low volume of released droplets allowed full evaporation of water during their flight towards the counter electrode without any need for drying gas or the addition of volatile solvents. The constructed device was successfully used for the deposition of water-free silver colloid without any requirement of its pretreatment. The deposition of completely dried nanoparticles on planar substrates eliminated undesirable coffee ring effect and deposits of increased homogeneity could be obtained. Electron microscopy confirmed no significant changes in the character of nanospray-processed nanoparticles. Finally, we also investigated several approaches for the improvement of the surface density of nanoparticles on the substrate at preserved time scale.
\end{abstract}

Keywords: Colloid, deposition, nanoparticles, nanospray, silver

\section{INTRODUCTION}

The deposition of nanostructures on the surface of a bulk material can significantly modify its properties and adjust it for target applications. The change in wettability or biological compatibility belongs to classical examples. Additionally, the localized nanoparticle deposits can act also as microelectrodes, substrates for surface-enhanced Raman spectrometry, or be part of sensing devices [1]. Most of these applications claim structures of uniform properties across the treated area. The full control of the deposition process is therefore an important prerequisite for its implementation into routine practice.

Commonly, the technology of inkjet printing or electrohydrodynamic printing is used for the highly-controlled depositions of colloids. In both cases, the experimental conditions do not allow evaporation of dispersing medium and the nanoparticles are deposited with a certain level of wetness (Figure 1). The amount of residual liquid highly influences the surface characteristics of the obtained structure (e.g. porosity). Moreover, significant differences in evaporation rates across the wet deposit give origin to an inhomogeneous distribution of the nanoparticles. This phenomenon is known as the coffee ring effect and challenges many procedures and protocols [2-4].

Electrospray has been proven as a potent competing tool for depositions of nanoparticles [1]. However, in many cases electrospray struggles with the residual presence of water too, which is typically addressed by long emitter-substrate distances, drying gases, or the addition of volatile solvents into a sprayed mixture. In this work, we used nano(electro)spray operated at a flow rate of $70 \mathrm{~nL} / \mathrm{min}$ only. Thus, the produced aerosol 
became extremely fine and the colloid reached the counter electrode completely dry without the necessity of any modification of colloid composition. The water-free deposition eliminates the risk of the coffee ring effect as well as the sintering of the deposited structures. Moreover, the nanospray-generated plume is considerably narrower in comparison to classical electrospray plume and allows precise positioning of the deposit at very low consumption of processed material.

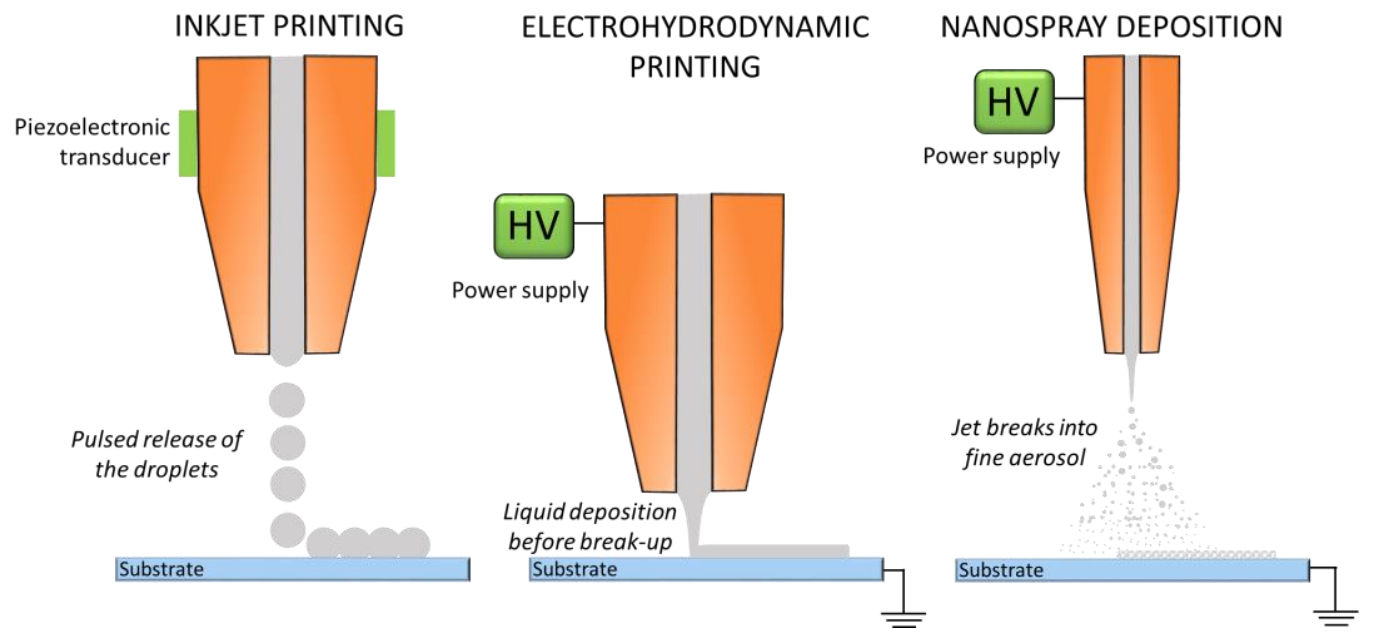

Figure 1 Schematic comparison of inkjet printing, electrohydrodynamic printing, and nanospray deposition of nanoparticles

Notably, the nanospray-assisted depositions sustain their remarkable simplicity of the instrumentation and no auxiliary drying components are needed. Thus, the operation of nanospray requires only a source of high voltage and continuous supply of sprayed mixture (typically a pump or pressurized gas) into an emitter. Due to this simplicity, such a set-up is barely commercially available and lab-made constructions have to be designed and constructed. Herein, we present a simple and compact yet very reliable set-up for nanospray production and we use it for the deposition of silver colloid on the substrate in a completely dried state.

\section{EXPERIMENTAL}

\subsection{Chemicals and methods}

The silver colloid synthesized by a Lee-Meisel protocol was used for nanospray-assisted depositions [5]. Its production is based on the reduction of silver nitrate (Sigma Aldrich) by sodium citrate (Lachema). In the standard protocol, the ratio of both reagents was approximately $1: 2(\mathrm{w} / \mathrm{w})$. The obtained dispersion was characterized via absorption spectrometry (UV-1800, Shimadzu) and scanning electron microscopy (Mira3, Tescan) both giving evidence of an average nanoparticle diameter in the range of $60-70 \mathrm{~nm}$.

We also used a modified protocol for silver nanoparticles synthesis. Therein, we reduced nanoparticles from silver nitrate using ten times excess of citrate $(\mathrm{w} / \mathrm{w})$. Such a colloid provided comparable characteristics as the colloid prepared according to the standard protocol.

\subsection{Instrumentation}

For a stable generation of nanospray aerosol, a special device was designed and fabricated. Two polysulfone parts mutually attachable via threading represent its key components. While one of the parts fixed a disposable vial with a colloid, the second part held an emitter and platinum electrode. Thus, only after the connection of both parts, the emitter and electrode immersed into a spray mixture and electrical connection was achieved. The threading sealed up the system tightly for the establishment of increased pressure via supplied nitrogen, 
which regulated a flow rate of the colloid through the immersed emitter. No significant pressure leakages were observed up to $6 \mathrm{Atm}$. However, for the generation of $70 \mathrm{~nL} / \mathrm{min}$, pressure of $0.35 \mathrm{Atm}$ was required only.

The emitter was fabricated from fused silica capillary (Polymicro Technologies) of an inner diameter of $25 \mu \mathrm{m}$. Its length of $28 \mathrm{~cm}$ allowed user-friendly manipulation. While one end of the emitter was immersed into a sprayed colloid, the opposite end was ground into a sharp tip on a lab-designed grinding device [6] and positioned in front of a grounded planar counter electrode with an attached substrate. Since the treated area is relatively small, a plastic mask was used for its easy localization. The emitter-substrate distance was $5 \mathrm{~mm}$, which provided reliably complete removal of water from aerosol during its flight towards the counter electrode. The optical camera (V160, Shenzhen Andonstar Tech Co.) monitored the behavior of the nanospray plume using the scattering of a green laser on the droplets of the aerosol. The described instrumentation is depicted in Figure 2.
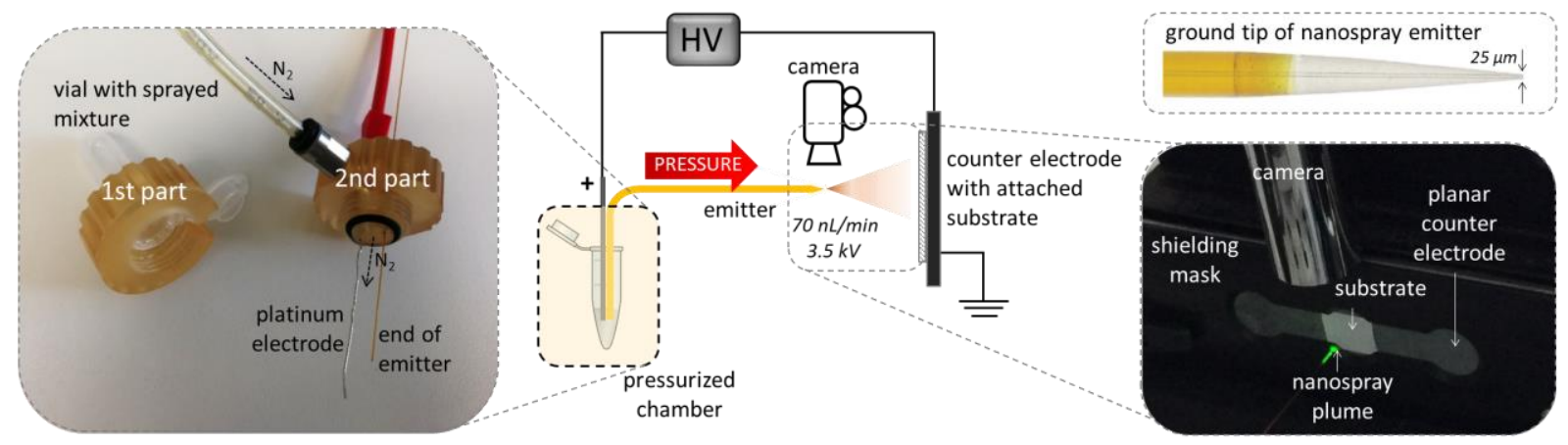

Figure 2 Schematic illustration and photographs of the developed set-up for nanospray-assisted deposition of nanoparticles on a planar substrate

\section{RESULTS AND DISCUSSION}

Deposits of nanostructures have got into the center of attention due to their unique properties useful in many cutting-edge devices [7-9]. The material of the processed colloid reflects the target application. Silver nanostructures belong to very popular dispersions due to their wide-range applicability and simplicity of their synthesis. In this work, we deposited via nanospray the silver colloid stabilized by molecules of citrate. The formation of a stable nanospray plume did not claim any colloid pretreatment and it could be reliably sprayed in its crude form. We determined a voltage of $3.5 \mathrm{kV}$ at a flow rate of $70 \mathrm{~nL} / \mathrm{min}$ as optimal conditions for long term performance. Within an hour of systematic monitoring, we did not observe any significant instabilities or changes in the plume character (Figure 3A).

The used low flow rate generated aerosol of very fine droplets. Their small volumes allowed fast evaporation of the dispersing medium at ambient conditions even at a short distance of $5 \mathrm{~mm}$. Thus, no components supporting the water evaporation were required and the instrumentation sustained its extraordinary simplicity and the drying was achieved only by the sufficient emitter-substrate distance. It should be also stressed, that the process of nanospraying did not change the overall character of the nanoparticles and that their shape and size were comparable with their state in the unprocessed colloid (Figure 3B). The absence of water in the deposited structures eliminated completely the coffee ring effect, which makes nanospray a very potent tool for the formation of evenly distributed deposits.

With respect to the uniform character of the deposit, one should consider a certain level of the size distribution of synthesized nanoparticles too. The effect of electrical field on the trajectory of nanoparticles is related with its size (i.e. mass), which might result preferential deposition of heavier entities in the central part of the target [10]. However, our microscopic observations did not reveal any significant central maximum in the surface density of nanoparticles. 
Further, the substrate-emitter distance influences also the treated area. At the emitter-substrate distance of $5 \mathrm{~mm}$ we treated round area with the estimated diameter of $\sim 8 \mathrm{~mm}$. While the increased gap would result in the larger spot and could be used for coating of extended area, at reduced distances the deposition of the nanoparticles becomes more precise. Additionally, very detailed patterns can be achieved using a fine stencil mask [11].

Although nanospray depositions bring a lot of benefits, the time of deposition procedure can significantly prolong due to the low volume flow rate. Therefore, we investigated possibilities of the reduction of deposition time via processing a colloid with five times increased concentration of nanoparticles.

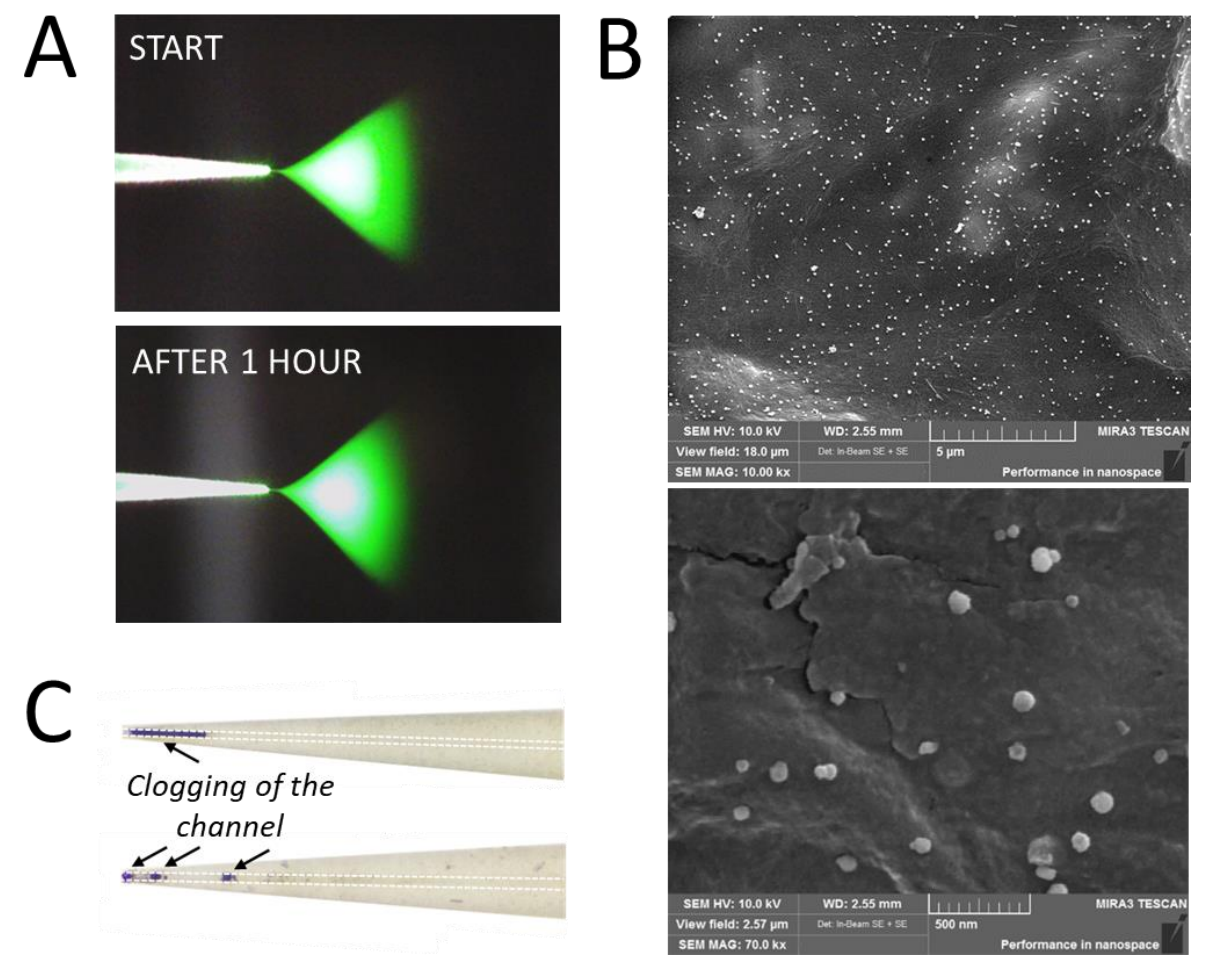

Figure $3 \mathrm{~A}$ - The comparison of nanospray plumes generated from silver colloid at the beginning of an experiment and after an hour. B - SEM figures of silver nanoparticles deposited on common office paper via nanospray. $\mathrm{C}-$ Two examples of emitters clogged by extensive structures created during the processing of silver colloid, which was concentrated five times by centrifugation.

Since the synthesis of such a colloid from its five times concentrated reagents (i.e., from solutions of silver nitrate and sodium citrate) would end up by the immediate formation of silver microstructures, we used for initial experiments a colloid synthesized by the standard protocol and concentrated it by centrifugation. Unfortunately, nanospraying of this dispersion resulted in a clogging of the emitter channel by extended structures created within several minutes (Figure 3C). To improve the stability of silver nanoparticles, we used for their reduction from silver nitrate approximately ten times excess $(\mathrm{w} / \mathrm{w})$ of citrate in the reaction mixture. This approach led to an excellent system providing a very stable performance of nanospray even in its centrifuge-concentrated form. Therefore, it represents a powerful possibility of the significant reduction of procedure time.

\section{CONCLUSION}

We proved that nanospray-assisted depositions of nanoparticles have great potential for the formation of evenly distributed structures. This can be achieved particularly due to low flow rates, which allow complete evaporation of water without the need for any complicated instrumentation. The increase of the concentration 
of stabilization agent in the silver colloid supports its stability and allows robust processing of concentrated dispersions and reduce the deposition time.

\section{ACKNOWLEDGEMENTS}

The authors gratefully acknowledge Czech Science Foundation for financial support (Grant No. 20 14069Y). Additional support was provided by Contripro a.s. company (Dolni Dobrouc, Czech Republic) and by Institutional support RVO 68081715 of Institute of Analytical Chemistry, Czech Academy of Sciences in Brno, Czech Republic.

\section{REFERENCES}

[1] JAWOREK, A., SOBCZYK, A.T., KRUPA, A. Electrospray application to powder production and surface coating. Journal of Aerosol Science. 2019, vol. 125, pp. 57-92.

[2] TANG, J., GOMEZ, A. Controlled mesoporous film formation from the deposition of electrosprayed nanoparticles. Aerosol Science and Technology. 2017, vol. 51, no. 6, pp. 755-765.

[3] CASTILLO, J. L., MARTIN, S., RODRIGUEZ-PEREZ, D., HIGUERA, F. J., GARCIA-YBARRA, P. L. Nanostructured porous coatings via electrospray atomization and deposition of nanoparticle suspensions. Journal of Aerosol Science. 2018, vol. 125, pp. 148-163.

[4] NOVAK, S., JOHNSTON, D. E., LI, C., DENG, W., Deposition of Ge23Sb7S70 chalcogenide glass films by electrospray. Thin Solid Films. 2015, vol. 588, pp. 56-60.

[5] LEE, P. C., MEISEL, D. Adsorption and surface-enhanced Raman of dyes on silver and gold sols. Journal of Physical Chemistry. 1982. vol. 86, no. 17, pp. 3391-3395.

[6] TYCOVA, A., PRIKRYL, J., FORET, F. Reproducible preparation of nanospray tips for capillary electrophoresis coupled to mass spectrometry using 3D printed grinding device. Electrophoresis. 2016, vol. 37, no. 7-8, pp. 924930.

[7] CUI, Z., HAN, Y., HUANG, Q., DONG, J., ZHU, YONG. Electrohydrodynamic printing of silver nanowires for flexible and stretchable electronics. Nanoscale. 2018, vol. 10, no. 15, pp. 6806-6811.

[8] AN, B. W., KIM, K., LEE, H., KIM, S. Y., SHIM, Y., LEE, D. Y., SONG, J. Y., PARK, J. U. High-resolution printing of 3D structures using an electrohydrodynamic inkjet with multiple functional inks. Advanced Materials. 2015, vol. 27, no. 29, pp. 4322-4328.

[9] HWANG, G. B., HEO, K. J., YUN, J. H., LEE, J. E., LEE, H. J., NHO, C. W., BAE, G. N., JUNG, J. H. Antimicrobial air filters using natural Euscaphis japonica nanoparticles. Plos One. 2015, vol. 10, no. 5.

[10] BROWN, N. A., ZHU, Y., GERMAN, G. K., YONG, X., CHIAROT, P.R. Electrospray deposit structure of nanoparticle suspensions. Journal of Electrostatics. 2017, vol. 90, pp. 67-73.

[11] KIM, J. W., YAMAGATA, Y., KIM, B. J., HIGUCHI, T. Direct and dry micro-patterning of nano-particles by electrospray deposition through a micro-stencil mask. Journal of Micromechanics and Microengineering. 2009, vol. 19 , no. 2 . 